TITLE:

\title{
Elongation/compaction of giant DNA caused by depletion interaction with a flexible polymer
}

$\operatorname{AUTHOR}(S):$

Kojima, Masahiro; Kubo, K.; Yoshikawa, Kenichi

\section{CITATION:}

Kojima, Masahiro ... [et al]. Elongation/compaction of giant DNA caused by depletion interaction with a flexible polymer. Journal of Chemical Physics 2006, 124(2): 024902.

\section{ISSUE DATE:}

2006-01-14

URL:

http://hdl.handle.net/2433/45685

\section{RIGHT:}

Copyright (2006) American Institute of Physics. This article may be downloaded for personal use only. Any other use requires prior permission of the author and the American Institute of Physics. 


\title{
Elongation/compaction of giant DNA caused by depletion interaction with a flexible polymer
}

\author{
M. Kojima, K. Kubo, and K. Yoshikawa ${ }^{\text {a) }}$ \\ Department of Physics, Graduate School of Science, Kyoto University, Kyoto, 606-8502, Japan
}

(Received 28 September 2005; accepted 2 November 2005; published online 10 January 2006)

\begin{abstract}
Structural changes in giant DNA induced by the addition of the flexible polymer Polyethylene Glycol (PEG) were examined by the method of single-DNA observation. In dilute DNA conditions, individual DNA assumes a compact state via a discrete coil-globule transition, whereas in concentrated solution, DNA molecules exhibit an extended conformation via macroscopic phase segregation. The long-axis length of the stretched state in DNA is about $10^{3}$ times larger than that of the compact state. Phase segregation at high DNA concentrations occurs at lower PEG concentrations than the compaction at low DNA concentrations. These opposite changes in the conformation of DNA molecule are interpreted in terms of the free energy, including depletion interaction. (C) 2006 American Institute of Physics. [DOI: 10.1063/1.2145752]
\end{abstract}

\section{INTRODUCTION}

It is well known that semiflexible polymers tend to segregate in the presence of nonadsorbing flexible polymers, through so-called depletion interaction. ${ }^{1-6}$ A schematic representation is shown in Fig. 1. We consider a flexible polymer that is much smaller than the persistence length ${ }^{7}$ of the semiflexible polymer. In this case, the semiflexible polymer can be treated as a hard cylinder at the scale of the flexible polymer and the flexible polymer is depleted from the region of a semiflexible polymer chain [Fig. 1(a)]. This region is called the depletion layer. When the semiflexible polymer segments come close to each other, the total depletion layer decreases and the volume occupied by flexible polymers increases. The presence of the flexible polymer causes an attractive force that arises from the entropy among the semiflexible polymer segments. The strength of this attractive force is identical regardless of intramolecular or intermolecular interaction, and only depends on the steric configuration of the interacting segments [Fig. 1(b)].

It has been reported that the formation of a liquidcrystalline phase from a concentrated solution of short DNA fragments can be induced by the addition of the flexible polymer Polyethylene Glycol (PEG), where DNA molecules are extended and aligned parallel, accompanied by macroscopic phase segregation. ${ }^{8-12}$ In contrast to the extension effect by depletion, it is also known ${ }^{13-16}$ that a compact state of DNA is generated by the addition of PEG. Thus, previous reports have indicated the opposite effects of a flexible polymer (either extension or compaction of DNA molecules), depending on the experimental conditions on the individual articles. The purpose of the present study was to obtain a comprehensive view of macroscopic phase segregation and the compaction of a semiflexible polymer by the addition of a flexible polymer.

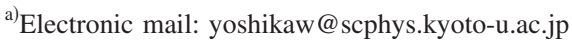

\section{EXPERIMENT}

Bacteriophage T4 DNA (166 kbp, contour length $57 \mu \mathrm{m})$ was purchased from Nippon Gene (Tokyo, Japan). The fluorescent dye Quinolinium, 1,19-[1,3-propanediylbis [(dimethyliminio)-3,1-propanediyl $]]$ bis $[4-[(3-$ methyl2(3H)-benzoxazolylidene)-methyl]]-, tetraiodide (YOYO-1) was obtained from Molecular Probes (Eugene, Oregon). PEG 6000 (average molecular weight $\cong 8200$ ) was obtained from Kishida Chemical Co., Ltd. (Osaka, Japan). Analytical-grade $\mathrm{NaCl}$ was obtained from Nacalai Tesque (Kyoto, Japan).

All procedures were performed at room temperature in Tris $100 \mathrm{mM}$ EDTA $10 \mathrm{mM}$ buffer (TE buffer $p \mathrm{H} \mathrm{8.0).} \mathrm{First,}$ PEG and $\mathrm{NaCl}$ were dissolved in a microtube. The concentration of PEG was adjusted as desired. The final concentration of $\mathrm{NaCl}$ was $100 \mathrm{mM}$ in all experiments. T4 DNA solution was mixed to give a final concentration of $20 \mu \mathrm{g} / \mathrm{ml}$ (A) or $0.1 \mu \mathrm{g} / \mathrm{ml}$ (B) in the microtube. Less than $1 \%$ of $\mathrm{T} 4$ DNA in (A) was stained with YOYO-1. This partially stained solution was mixed with a solution free from dye or solutions that had already been equilibrated with dye. In contrast, the T4 DNA molecules in (B) were uniformly stained. The ratio between labeled DNA and fluorescent dye was [labeled DNA(bp) $] /[$ YOYO-1] $=5$ in molar units. It has been confirmed that the contour length and persistence length remain essentially constant, i.e., the effect of dye staining is negligible. ${ }^{17}$ The (A) samples were gently shaken and allowed to stand for a few hours. After this rest period, the samples were gently vortexed. The (B) samples were allowed to stand for $24 \mathrm{~h}$ after mixing.

Fluorescent microscopic images of DNA and polarization microscopic images of the same regions were observed using a BX 60 (Olympus, Japan) fluorescence and polarization microscope with a color charge-coupled device (CCD) camera (Watec, Japan). The fluorescence images of the DNA chain were observed using an Axiovert 200 fluorescence microscope (Carl Zeiss, Germany) with a high-resolution EBCCD camera (Hamamatsu, Japan). 

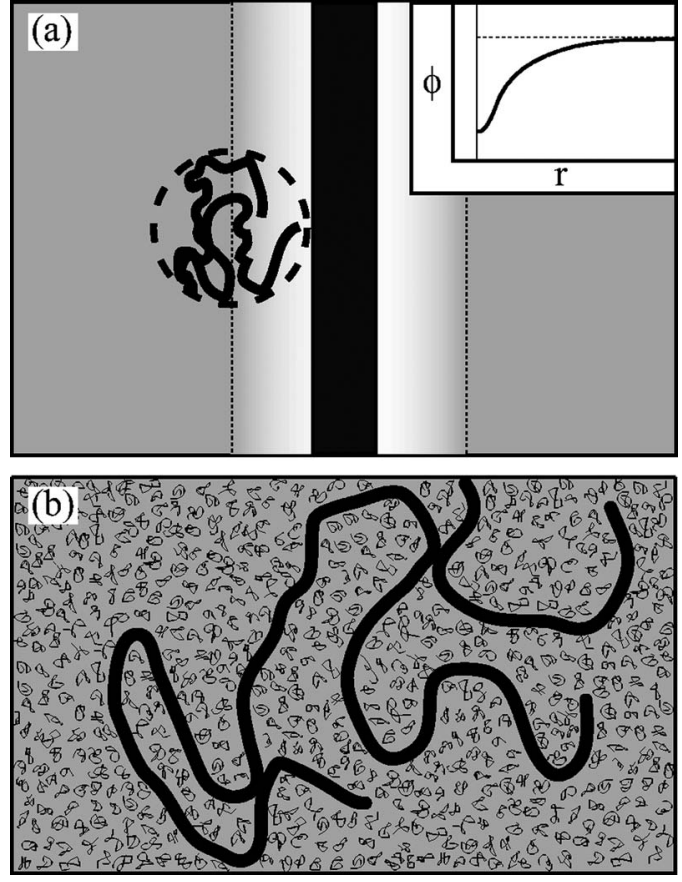

FIG. 1. Schematic representation on the mixed solution of semiflexible and flexible polymers. (a) Microscopic view on a flexible polymer chain around a semiflexible polymer. Near the surface of the semiflexible polymer, the volume fraction of flexible polymer is lower than that in the bulk. Inset: schematic density profile of the flexible polymer, where $\phi$ is the volume fraction of the flexible polymer and $r$ is the distance from the surface of the semiflexible polymer. (b) Coarse-grained view on the conformation of a semiflexible polymer in the solution of flexible polymer chains.

\section{RESULTS AND DISCUSSION}

Figure 2 shows typical fluorescence images of individual DNA molecules. At a low PEG concentration, DNA molecules assume a random coil state regardless of the DNA concentration [Figs. 2(a) and 2(d)]. With an increase in the PEG concentration, DNA molecules at a high DNA concentration show an elongated conformation [Fig. 2(b)], accompanied by macroscopic phase segregation between PEG-rich and DNA-rich phases. The long-axis length of an elongated
DNA chain approximately corresponds to its contour length; in Fig. 2(b), the full length is about $50 \mu \mathrm{m}$. Figure 2(c) shows a polarization microscopy image of the same region as in Fig. 2(b). The DNA molecule in (B) assumes a compact state through the coil-globule transition [Fig. 2(e)]. Note that the long axis of an elongated DNA chain is about $10^{3}$ times larger than that in the compact state.

Figure 3(a) shows the dependence of the volume fraction of DNA $\phi_{\mathrm{DNA}}$ inside the generated DNA-rich phase on the PEG concentration. The lowest PEG concentration that induced phase segregation was $180 \mathrm{mg} / \mathrm{ml}$ [Fig. 3(a)]. The volume fraction of DNA $\phi_{\text {DNA }}$ inside the generated DNArich phase appears to increase with an increase in the PEG concentration. The line in Fig. 3(a) is fitted by the leastsquares method with a PEG concentration within the range of $180-230 \mathrm{mg} / \mathrm{ml}$ and the gradient is ca.0.002. In the experiments, the actual volume of the DNA-rich phase in the phase-separated solution was measured. Then, $\phi_{\mathrm{DNA}}$ was calculated based on the assumption that all of the DNA molecules are transferred to the DNA-rich phase, togther with the basic DNA property (diameter $d=2 \mathrm{~nm}$, contour length $L=57 \mu \mathrm{m})$.

Figure 3(b) shows the distribution of the long-axis length of DNA molecules through the coil-globule transition versus the PEG concentration. The closed and open circles show the average long-axis length of DNA molecules in the coil and globule states, respectively. DNA molecules in both compact and coiled states were observed at a PEG concentration of around $210 \mathrm{mg} / \mathrm{ml}$ and the number of DNA molecules in the compact state was approximately twice that in the coil state.

Note that the critical PEG concentration for phase segregation was lower than that for the coil-globule transition. This result means that the elongated state of DNA molecules is more stable than the compact state if the DNA concentration is high enough.

Let us discuss both transitions in terms of free energy. The total free-energy variance per DNA chain $\Delta F_{\text {tot }}$ can be written as
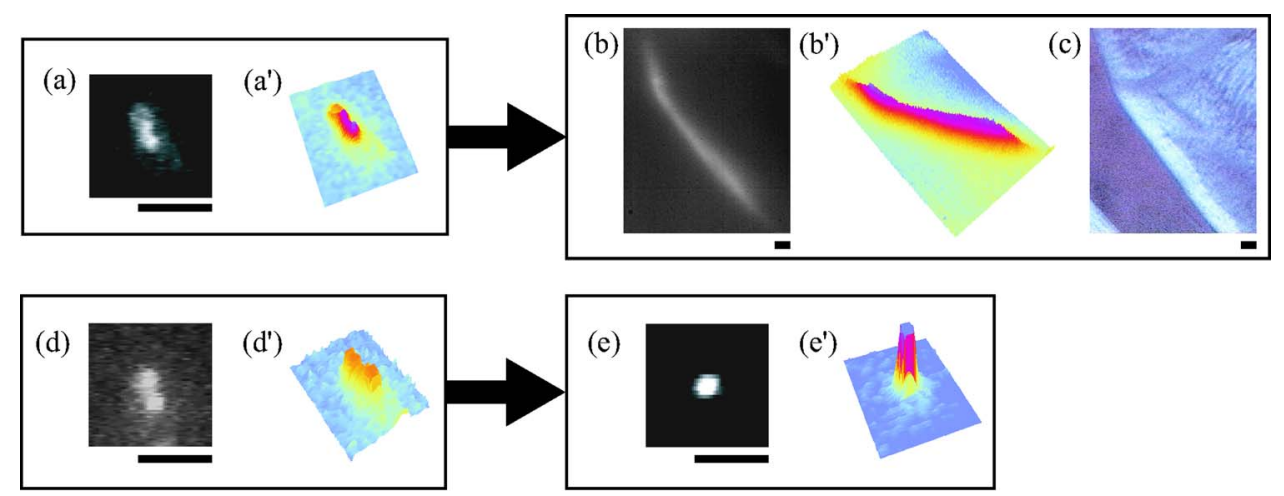

FIG. 2. (Color online) Conformational change of single DNA by the addition of PEG. All scale bars represent $3 \mu \mathrm{m}$. (a) Selectively stained DNA molecule with a coiled conformation in concentrated DNA solution (DNA $20 \mu \mathrm{g} / \mathrm{ml}$, PEG $170 \mathrm{mg} / \mathrm{ml}, \mathrm{NaCl} 100 \mathrm{mM}$ ). (b) Selectively stained DNA molecule with a stretched conformation in a liquid-crystalline phase, under the condition of phase segregation between a DNA-rich phase and a PEG-rich phase (DNA $20 \mu \mathrm{g} / \mathrm{ml}$, PEG $230 \mathrm{mg} / \mathrm{ml}, \mathrm{NaCl} 100 \mathrm{mM}$ ). (c) Polarization microscopy image on the same region as in (b). (d) Coiled DNA molecule in dilute DNA solution (DNA $0.1 \mu \mathrm{g} / \mathrm{ml}$, PEG $170 \mathrm{mg} / \mathrm{ml}$, and $\mathrm{NaCl} 100 \mathrm{mM}$ ). (e) Compact DNA in dilute DNA solution (DNA $0.1 \mu \mathrm{g} / \mathrm{ml}, \mathrm{PEG} 230 \mathrm{mg} / \mathrm{ml}, \mathrm{NaCl} 100$ $\mathrm{mM}) .\left(\mathrm{a}^{\prime}\right),\left(\mathrm{b}^{\prime}\right),\left(\mathrm{c}^{\prime}\right)$, and $\left(\mathrm{d}^{\prime}\right)$ show fluorescence-intensity distributions for (a), (b), (c), and (d), respectively. 
(a)

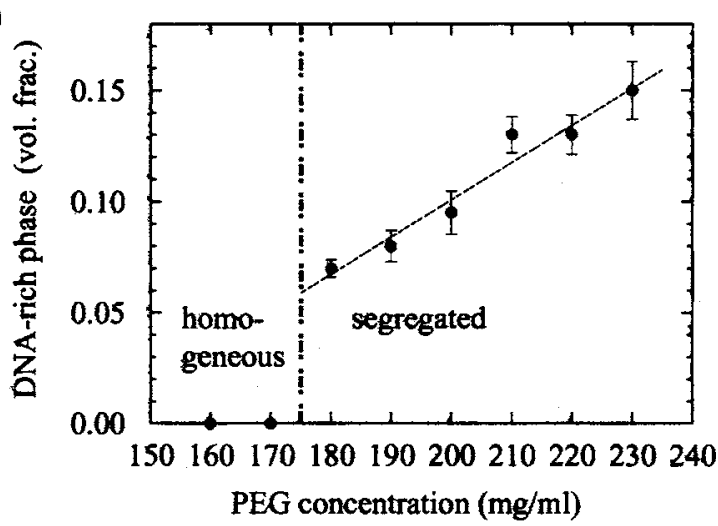

(b)

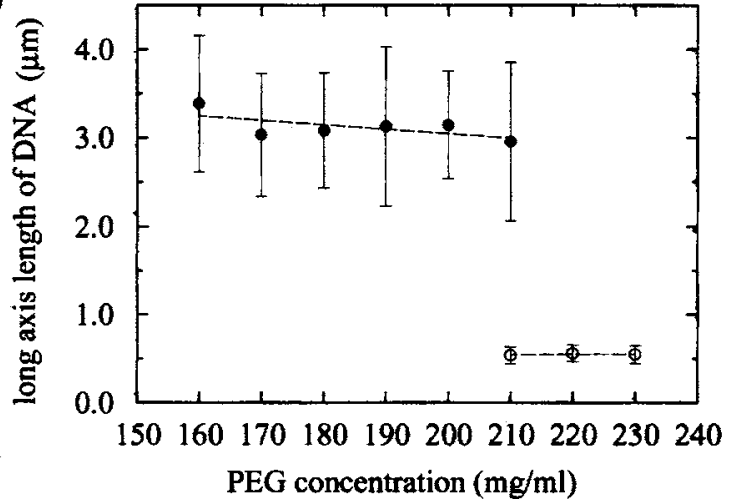

FIG. 3. (a) Phase behavior of DNA-PEG solution represented as the volume fraction of the DNA-rich phase, the zero volume fraction corresponds to a homogeneous solution (T4 DNA $20 \mu \mathrm{g} / \mathrm{ml}, \mathrm{NaCl} 100 \mathrm{mM}$ ). Below $170 \mathrm{mg} / \mathrm{ml}$ of PEG, the system exhibits a homogeneous state. Above $180 \mathrm{mg} / \mathrm{ml}$ of PEG, a liquid-crystalline DNA phase was induced accompanied by phase segregation. (b) Conformational change of single DNA. The dependence of the average long-axis length is shown together with the standard deviation (T4 DNA $0.1 \mu \mathrm{g} / \mathrm{ml}, \mathrm{NaCl} 100 \mathrm{mM}$ ). The coiled and compact states of DNA molecules coexisted at $210 \mathrm{mg} / \mathrm{ml}$ of PEG.

$$
\Delta F_{\mathrm{tot}} \cong \Delta F_{\mathrm{dp}}+\Delta F_{\mathrm{el}}
$$

where $\Delta F_{\mathrm{dp}}$ describes the gain in the decrease in the depletion layer for PEG per DNA chain. $\Delta F_{\mathrm{el}}$ describes the elastic part of the free energy for a single DNA chain. For simplicity, the electrostatic and van der Waals interactions among DNA, PEG, and ions are neglected in Eq. (1). These miscellaneous effects are considered to be incorporated in the effective elastic energy.

$\Delta F_{\text {dp }}$ can be written approximately as ${ }^{7,18}$

$$
\Delta F_{\mathrm{dp}} / k T=\frac{-\Delta V}{d^{3}}\left(\frac{\phi_{\mathrm{PEG}}}{N_{\mathrm{PEG}}} \ln \frac{\phi_{\mathrm{PEG}}}{N_{\mathrm{PEG}}}-\frac{\phi_{\mathrm{PEG}}}{N_{\mathrm{PEG}}}\right) \quad(<0),
$$

where $T$ is temperature, $k$ is the Boltzmann constant, $\Delta V(<0)$ is the variation in the depletion layer for PEG around a DNA molecule, $d$ is the elementary spacing in the Flory-Huggins model which was defined to be equal to the width of the DNA Kuhn segment, $\phi_{\mathrm{PEG}}$ is the volume fraction of PEG, and $N_{\mathrm{PEG}}$ is the average degree of polymerization of PEG molecules. In Eq. (2), the translational entropy of water is neglected, since the effective volume occupied by water may not change greatly. We also treat $\phi_{\mathrm{PEG}}$ as a constant through the transitions because $\Delta V$ is much smaller than the total volume of the system.

$\Delta V$ may be approximately given as ${ }^{2}$

$$
\Delta V \cong \pi L\left(\frac{d}{2}\right)^{2} / \phi_{\mathrm{DNA}}-\pi L\left(\frac{d}{2}+R g_{\mathrm{PEG}}\right)^{2}
$$

where $L$ is the contour length of a DNA chain and $R g_{\text {PEG }}$ $=\sqrt{N_{\mathrm{PEG}}^{2 \nu} b^{2} / 6}$ is the radius of gyration of a PEG molecule $(\nu$, Flory exponent; $b$, PEG monomer size). The first term on the right-hand side is the volume occupied by a DNA molecule after the transition. The second term represents the depletion layer for the center of mass of PEG around a DNA molecule before the transition. For analytical treatment, the depletion layer before the transition is represented as a step function, although the actual depletion layer will not be so simple because of the continuous PEG concentration profile.

Following Ref. 7, the elastic free energy $F_{\text {el }}$ asymptotically reads

$$
\begin{aligned}
& \Delta F_{\mathrm{el}} / k T \cong \frac{3}{2}\left(\alpha^{2}+\alpha^{-2}\right), \\
& \alpha=\frac{R_{\mathrm{DNA}}}{N_{\mathrm{DNA}}^{1 / 2} l},
\end{aligned}
$$

where $\alpha$ is the expansion factor of the DNA coil with respect to its ideal size, $R_{\mathrm{DNA}}$ is the long-axis length of a DNA molecule, $N_{\mathrm{DNA}}$ is the number of Kuhn segments, and $l$ is the Kuhn length of a DNA chain.

In the case of compaction, $\Delta F_{\text {el }}$ can be written as $\Delta F_{\text {tr }}$ which is a toroidal shape of compact DNA. ${ }^{19}$

$$
\Delta F_{\mathrm{tr}} / k T \cong \frac{l L}{\left(R_{\mathrm{DNA}} / 2\right)^{2}}
$$

TABLE I. Change in free energy accompanied by the transition of coiled DNA into elongated/compact states.

\begin{tabular}{lcccccc}
\hline \hline & $\Delta V\left(\mathrm{~nm}^{3}\right)^{\mathrm{a}}$ & $\phi_{\mathrm{PEG}}^{\mathrm{b}}$ & $\Delta F_{\mathrm{dp}}(k T)^{\mathrm{c}}$ & $R_{\mathrm{DNA}}(\mathrm{nm})^{\mathrm{d}}$ & $\Delta F_{\mathrm{el}}(k T)^{\mathrm{e}}$ & $\Delta F_{\mathrm{tr}}(k T)^{\mathrm{f}}$ \\
\hline Elongation & $-1.2 \times 10^{6}$ & 0.14 & -900 & $5 \times 10^{4}$ & +700 & $\ldots$ \\
Compaction & $-3.6 \times 10^{6}$ & 0.16 & -3000 & $70^{14}$ & +2000 & +2300 \\
\hline \hline
\end{tabular}

${ }^{\mathrm{a}}$ Volume change in the depletion layer for PEG around a DNA molecule.

${ }^{b}$ Volume fraction of PEG.

${ }^{c}$ Free-energy change owing to the depletion interaction per single DNA chain.

${ }^{\mathrm{d}}$ Long-axis length of DNA after the transition.

${ }^{\mathrm{e}}$ Free-energy change of elastic term single DNA chain, estimated from the volume change.

${ }^{\mathrm{f}}$ Free-energy change of elastic term by the consideration of toroidal compact state in DNA (Ref. 19). 
In the present paper, the constants are set as $d=2 \mathrm{~nm}$, $b=0.38 \mathrm{~nm},{ }^{20} \nu=0.6, N_{\mathrm{PEG}}=186$, and $L=57 \mu \mathrm{m}$. From Eqs. (2), (4), and (5), $\Delta F_{\mathrm{dp}}, \Delta F_{\mathrm{el}}$, and $\Delta F_{\text {tr }}$ are calculated, as shown in Table I. In the case of macroscopic phase segregation, $\Delta V$ was $-1.2 \times 10^{6} \mathrm{~nm}^{3}$, the critical PEG concentration was $180 \mathrm{mg} / \mathrm{ml}\left(\phi_{P}=0.14\right)$ and the volume fraction was $\phi_{\mathrm{DNA}} \cong 0.07$ from Fig. 3 . Therefore, $F_{\mathrm{dp}}$ was calculated to be about $-900 \mathrm{kT}$. Since $R_{\mathrm{DNA}}$ was about $5 \times 10^{4} \mathrm{~nm}, \Delta F_{\text {el }}$ is evaluated to be about $+700 \mathrm{kT}$. In the coil-globule transition, it has been determined by the measurement of the hydrodynamic radius ${ }^{16}$ and a molecular-dynamics (MD) simulation ${ }^{21}$ that the compact state of a DNA molecule is completely packed. Thus, we consider $\phi_{\mathrm{DNA}} \cong 1$ to be a good approximation. Thus, $\Delta V$ is calculated as $-3.6 \times 10^{6} \mathrm{~nm}^{3}$ and the critical PEG concentration is $210 \mathrm{mg} / \mathrm{ml}\left(\phi_{P}=0.16\right) . \Delta F_{\mathrm{dp}}$ is estimated to be $-3 \times 10^{3} \mathrm{kT}$. Based on electron-microscopic observation, ${ }^{14}$ the long-axis length of toroidal DNA is roughly $70 \mathrm{~nm}$. Consequently, $\Delta F_{\mathrm{el}}$ and $\Delta F_{\text {tr }}$ are deduced to be +2000 and $+2300 \mathrm{kT}$, respectively. Despite this simple estimation, it becomes clear that the gain in free energy involving PEG and the loss involving the DNA conformation are on the same order in both transitions. In other words, the above discussion indicates that $\Delta F_{\mathrm{dp}}$ and $\Delta F_{\mathrm{el}}$ mainly contribute to the transitions and direct "attractive" chemical interaction between PEG and DNA is not so important.

In the present consideration on the free energy, we have not discussed the effect of small salt ions. Actually, the effect of the translational entropy of counterions has been indicated in the case of compaction. ${ }^{22}$ It has also been indicated that salt ion promotes the DNA compaction in PEG solution. ${ }^{18}$ As a next step, it may be of interest to compare the effect of salt between dilute DNA and concentrated DNA solutions in the presence of PEG.

In summary, we studied both the segregation and compaction of giant DNA induced by depletion interaction. There is a marked difference in the critical concentrations of flexible polymer needed to cause these transitions and the essence of this difference can be interpreted in terms of the change in free energy with depletion interaction.

\section{ACKNOWLEDGMENT}

This work was supported by a Grant-in-Aid for Scientific Research in Priority Areas "System Cell Engineering by Multi-scale Manipulation" (Grant No. 17076007) from the Ministry of Education, Culture, Sports, Science and Technology of Japan.

${ }^{1}$ S. Asakura and F. Oosawa, J. Chem. Phys. 33, 183 (1954).

${ }^{2}$ S. Asakura and F. Oosawa, J. Polym. Sci. 33, 183 (1958).

${ }^{3}$ J. F. Joanny, L. Leibler, and P. G. D. Gennes, J. Polym. Sci., Polym. Phys. Ed. 17, 1073 (1979).

${ }^{4}$ T. C. Lubensky, Solid State Commun. 102, 187 (1997).

${ }^{5}$ R. R. Netz and D. Andelman, Phys. Rep. 380, 1 (2003).

${ }^{6}$ R. Tuinier, J. Rieger, and C. G. de Kruif, Adv. Colloid Interface Sci. 103, 1 (2003).

${ }^{7}$ A. Y. Grosberg and A. R. Khokhlov, Statistical Physics of Macromolecules (AIP, New York, 1994).

${ }^{8}$ C. F. Jordan, L. S. Lerman, and J. Venable, Nature New Biol. 236, 67 (1971).

${ }^{9}$ F. Livolant and A. Leforestier, Prog. Polym. Sci. 21, 1115 (1996).

${ }^{10}$ H. H. Strey, V. A. Parsegian, and R. Podgornik, Phys. Rev. Lett. 78, 895 (1997).

${ }^{11}$ M. Leonard, H. Hong, and H. H. S. N. Easwar, Polymer 42, 5823 (2001).

${ }^{12}$ H. M. Harreis, A. A. Kornyshev, C. N. Likos, H. Löwen, and G. Sutmann, Phys. Rev. Lett. 89, 018303 (2002).

${ }^{13}$ L. S. Lerman, Proc. Natl. Acad. Sci. U.S.A. 68, 1886 (1971).

${ }^{14}$ U. K. Laemnli, Proc. Natl. Acad. Sci. U.S.A. 72, 4288 (1978).

${ }^{15}$ K. Minagawa, Y. Matsuzawa, K. Yoshikawa, A. R. Khokhlov, and M. Doi, Biopolymers 34, 555 (1994).

${ }^{16}$ K. Yoshikawa and Y. Matsuzawa, Physica D 84, 220 (1995).

${ }^{17}$ N. Yoshinaga, T. Akitaya, and K. Yoshikawa, Biochem. Biophys. Res. Commun. 286, 264 (2001).

${ }^{18}$ V. V. Vasilevskaya, A. R. Kohokhlov, Y. Matsuzawa, and K. Yoshikawa, J. Chem. Phys. 102, 6595 (1995).

${ }^{19}$ J. Ubbink and T. Odjik, Europhys. Lett. 33, 353 (1996).

${ }^{20}$ D. Marsh, Biophys. J. 86, 2630 (2004).

${ }^{21}$ H. Noguchi, S. Saito, S. Kidoaki, and K. Yoshikawa, Chem. Phys. Lett. 261, 527 (1996).

${ }^{22}$ T. Saito, T. Iwaki, and K. Yoshikawa, Europhys. Lett. 71, 304 (2005). 